\title{
Technological Problems Associated with Subcutaneous Microchips for Human Identification (SMHId)
}

\author{
Silvia Covacio \\ University of South Australia, Adelaide, Australia
}

\author{
Silvia.Covacio@unisa.edu.au
}

\begin{abstract}
Past technological developments have encouraged visionary information systems and created fresh linkages that transcend disciplinary boundaries. For example, the long-term research of animal identification technology has paved the way for the instigation and possible widespread implementation of SMHId.

Unfortunately, the SMHId system does not come without its own social problems requiring a proactive approach. One such problem under scrutiny is the upsurge of satellite communication technology using microwave radio frequency and related technological gadgets. Past and current research of microwave radiation indicates there are detrimental effects for the environment and humans. If an open environment SMHId configuration is instigated and utilizes global positioning system (GPS) satellite technology, the increase in health problems will consequentially increase pressure on health services already under economic duress.
\end{abstract}

Keywords : SMHId, subcutaneous microchips, microwave radio frequency, GPS, satellite, health issues, health services

\section{Introduction}

Allow a liberal application of Bogard's (1996) "social science fiction" in the following possible future scenario ...

A person wakes at a set time, not by the buzz of an alarm clock but through the release of stimulants into the blood stream. The controlled release of stimulant is monitored by a subcutaneous microchip similar to those currently available for insulin release in diabetics (Quan 1999, Weber 2000). The microchip has a unique code identifying each individual microchip and therefore the person. Shopping, banking, and logging onto the computer network to begin work or study is as simple as a 'flick of the wrist', (one of many locations a SMHId could be injected). Data collected from the SMHId system is constantly uploaded to marketing supercomputers that analyze input to find trends, monitor economies, and target individuals with specific advertisements tailored to meet personal demographics revealed from the collated data. The specialized computer system monitoring the SMHId recipient at home is connected via the Internet, which uses GPS technology and microwave earth stations to transfer data.

Material published as part of these proceedings, either on-line or in print, is copyrighted by Informing Science. Permission to make digital or paper copy of part or all of these worksfor personal or classroom use is granted without fee provided that the copies are not made or distributed for profit or commercial advantage AND that copies 1) bear this notice in full and 2) give the full citation on the first page. It is permissible to abstract these works so long as credit is given. To copy in all other cases or to republish or to post on a server or to redistribute to lists requires specific permission from the publisher at Publisher@InformingScience.org
This scenario is not unlikely; it reveals the technically advanced social system within which we live. The main difference is our current use of magnetic-striped cards, and tags, with the added security of personal identification numbers, passwords, or biometric identifiers allowing access to separate information systems within our daily lives. The above scenario introduces implantable 
microchips as an alternative to other human identification devices.

SMHId is an encompassing generic term specifically applied to subcutaneous microchips for human identification systems, clearly distinct from microchips for animal identification described as 'transponders' in the veterinary literature. The term 'transponder' is also utilized in radio frequency identification (RFID) literature to describe any tagging device based on microchip technology (Marsh 2003), and in data communication literature, in relation to a single satellite operating a number of frequency bands, also called 'transponder channels' (Stallings and van Slyke 1994). The SMHId acronym has been created and used to isolate and differentiate this very specific human identification system from related areas and disciplines. The term SMHId also includes current and future companies in the SMHId supply chain. Using registered names for SMHId, exclusively targets the identified company, thereby excluding all other manufacturers and distributors of SMHId and related products. Also, the abbreviation adheres to terminology standards in information technology and systems (ITS), and past research shows a lack of specific terminology applied universally to this artefact.

Developments in the area of communications, networking, and surveillance systems continue to occur globally. Many business are driven by economic pressures and lured by the seductive profitability of information gathering systems, and participate in the sale of business and personal information on the information market. E-commerce, e-banking, and e-shopping are slowly gaining community acceptance as social, economic, and technological factors impel adoption of the technology. Information gathering is intrinsically part of those systems and the large amount of data transfer requires utilization of broadband and GPS technologies.

The increased flow of data traffic has encouraged the instigation of broadband telecommunication systems, and a number of broadband systems are being developed. For example, a low earth orbit (LEO) broadband satellite system being instigated is Teledesic, and marketed as the "Internet-in-the-Sky" (www.teledesic.com). If SMHId is utilized in conjunction with "Internet-in-the-Sky" and other interconnected GPS systems, the capabilities to track SMHId recipient's every movement, and every financial transaction, establishes a global surveillance and economic system.

ITS capacity to collate personal information causes many people to be increasingly concerned with issues such as ethics, privacy, surveillance, power, and control. These and other related issues are being addressed by a number of academics but discourse in these areas will not solve the most basic problem biologically safe GPS-based data transfer. The technology being utilized in GPS satellites, and the possible social consequences of using GPS satellites with SMHId will possibly impact many areas. This paper explores the possible technological problems and the influence of those problems on individual wellbeing, and health systems.

Research in the field of microwave radio frequency and magnetic fields, also described as non-ionizing radiation, has produced numerous conflicting reports and articles on the causal effects of microwave and radio frequency radiation (Australian Communications Authority 2000, Bloomfield 2002, Inquiry into Electromagnetic Radiation 2001, Tomljanovic et al 1997). If the technology is dangerous, and industryfavored academics and industry advocates are suppressing information because it will impact on the economic viability and management of broadband and GPS, the detrimental causal effects could generate an evolutionary selection process favoring those opposed to SMHId and related technology.

\section{Evolution of SMHId}

Decisions made by our ancestors continue to effect changes that we are experiencing in our social structures today. There are many previously published articles in journals and texts regarding the development and application of technology from an historical perspective including the miniaturization of technological components. Technology has not only stimulated and accelerated further technological devel- 
opment, but Giddens (1993) states that technology has influenced society's development - a soft technological deterministic perspective; an argument that has been discussed elsewhere.

In society's development, the use of labeling or tagging items assists identification, classification, and control of artefacts. Clarke (1994) discusses the various techniques used to identify people including names or other physical peculiarities that have served as an identification aid for individuals or social groups, and commodities were commonly tagged with the name or other symbolic identifier. The use of identification became important when loss or theft of the item or goods impaired economic flow to the individual or group. The history of tagging commodities includes the identification of animals, and unfortunately, other humans.

Rossing (1999) points out two successful tagging features; ease of attaching the tag to the commodity, and tag permanence, and continues to explain numerous identification methods and how the increased use of ITS assisted the growth of identification technology based on microchips. Experimentation using microchips in external ear tags began in early 1970's, assisting animal identification in large herds. Further miniaturization of electronic components in the 1980's produced passive and programmable injectable microchips for animals and hand-held readers to scan for and read the unique microchip code.

History reveals new products, techniques or ideas, such as, pharmaceutical products, surgical techniques, and the initial space exploration, are often tested on animals prior to human trials and wider market implementation: animal experimentation often leads to human implementation. This is also true with SMHId. In 1993, Gruys, Schakenraad, Kruit and Bolscher validated in vitro bio-compatibility of subcutaneous microchips with human tissue and blood. Lipomatrix Incorporated (Knapp and Belsey 1998), an early adopter of SMHId ideas and technology, registered a patent in the United States with the following details,

\begin{abstract}
"A passive transponder may be encoded with a binary number or code of 64 bits or more and then associated with or mounted to virtually any implant intended for implantation in a human including temporary implants such as drug release implants and organ displacement devices. After implantation, the transponder's code may be conveniently read with a hand-held electromagnetic reader which may merely be brought within proximity of the transponder."
\end{abstract}

The number of patents utilizing subcutaneous microchips has risen significantly, because of the success of biomaterial compatibility minimizing possible long term affects for humans (Pugh 1998). Biomaterials must now adhere to strict and specific safety standards for human use.

However, research in radio frequency identification (RFID) and mobile telephone technology has revealed a growing concern with the effects of radio frequency and non-ionizing radiation on organic matter. It has been revealed a number of low-level, and possible high-level risks are associated with the use of radio frequency technology. Effects of X-rays and gamma rays have been well documented in medical and electronic journals, and this article does not deal with those specific frequencies. This article deals with the band of radio frequency labeled in journals and texts as the 'microwave' spectrum.

\title{
Possible Technological Problems
}

Acceptance of identification systems using electronic tagging is rapidly increasing. This is evident from a large number of fields utilizing microchip technology, for example, motor vehicle manufacturing, various commercial transport systems, distribution and warehouse systems, consumer products, intelligent highway systems, security, and access systems. Many electronic tagging systems rely on GPS to track and uniquely identify artefacts, animals, and in recent years, people.

In this discourse, the identified technological problems relate to a number of issues; the physical artefact - the microchip itself and any metal components, telecommunication technologies specifically microwave radio frequency, and the social consequences of the technology with specific focus on health issues. 


\section{Microchip Issues}

Past research on transponders for animals has revealed limitations including; transponder distance for accurate readings of microchip code (Sorensen, Buss and Tyler 1995); physical size and pliability of the microchip (Economist Technology Quarterly 2001); and, tissue impact around the injection site and migration of the microchip under the skin (Lambooij, Langeveld, Lammers and Huiskes 1995). Carcinogenicity and toxicity were initially an issue with metal ear tags in animals (Rao and Edmonson 1990) and this seminal work guided further research in biomaterial for medical implants, and is quoted in literature with regards to transponder for animals in relation to the animal's welfare (Appell 2003, Park and Weiser 1998). Biomaterials are now manufactured from materials with minimal toxicity to animals and humans.

A related health concern to the physical artefact is the possible rejection of the SMHId due to an allergic reaction. The animal research data records a very low rejection rate or allergic reactions to transponders; approximately 1 - 2\% (Gruys et al 1993, Lambooij et al 1995). However, humans are becoming increasingly environmentally intolerant and a greater number of people suffering allergies is being reported by Disability Online (2002), Woolcock Institute of Medical Research (2002) and the National Institute of Allergy and Infectious Diseases (2002). This may indicate higher reaction and rejection rates to SMHId than previously expected.

The physical attributes of certain types of subcutaneous microchips may be an issue especially if the microchip has metal components. The magnetic fields produced by radio frequency can heat metal objects, as reported by Department of Health, Ed ucation, and Welfare (1973), and Smith et al (1999), and may cause localized heat lesions. The research describes metallic medical implants that could heat or burn flesh when radio frequency fields cause a temperature rise in wires. A situation that may have impact in this regard, is the possible development of a new type of SMHId based on a combination of an external battery powered GPS tracked artefact, similar to mobile telephone technology, and the minute passive microchip. The miniaturization of the larger components allows for creation of a SMHId with a small rechargeable battery for simple implantation and GPS tracking capabilities. This would be detrimental to SMHId recipients because of the problems directly associated with microwave radio frequency, described in the next section, and secondly, an increase in health problems impacting a failing health system.

\section{Telecommunication Technology and Microwave Frequency}

Satellite or earth stations using microwave radio frequency has been successfully utilized since the early 1960's (Leick 1995) and continue to expand in use throughout many communities for various telecommunications needs. Microwaves are used to transfer data to and from earth stations, and satellites using point-to-point or broadcast configurations in the band range of 2 - $40 \mathrm{GHz}$ (Stallings and van Slyke 1994), can occupy three orbits (3G-Generation 2000). A high geosynchronous earth orbit (GEO) at approximately 21,000 miles or 35,000 kilometers, a medium earth orbit (MEO), and a low orbit (LEO) between 180 - 1056 miles or 290 - 1700 kilometers (3G-Generation 2000, Stallings and van Slyke 1994).

GEO satellites require longer signal time and an intense transmission due to the distance from earth and a thicker layer of atmosphere to penetrate. Leick (1995) explains that two signals are required to ensure each data transmission is successful. He indicates that the purpose of the second frequency in GPS is to neutralize the affect of the ionosphere on signal propagation. This clearly indicates successful microwave signals effectively double the radio frequency radiation exposure to the environment and humans. Research and implementation of LEO's provides a partial solution to this problem due to their closeness to the earth, taking less data transfer time, less signal intensity required, and less atmosphere to penetrate. (See Figure 1 for microwave satellite fundamentals.) 


\section{Microwave Radiation within Earth's Atmosphere} Silvia Conocio August 2002

Earth's Atmosphere - protects Earth from harmful solar and space radiation including ultraviolet and microwave light waves. Atmosphere is approx 400 miles thick.

\section{CONCERNS:}

Microwave ovens used to heat and cook food are considered dangerous if radiation leaks from a faulty oven. The frequency used in microwave ovens is comparable to the frequency of microwave communication satellites.

Microwaves heating up the atmosphere will affect all individuals even those who are not utilising micro wave communication systems.

Another possible cause of global warming?

Communication satellites using microwaves to transfer data to earth stations using point-to-point or broadcast configurations with common frequencies ranging from $2-40 \mathrm{GHz}$ at high intensity to penetrate through layers of atmosphere.

Satellites maintain 3 orbits: high at approx 21,000 miles $(35,000 \mathrm{~km})$, medium, and low at 435 miles $(700 \mathrm{~km})$.

$\mathrm{NB}$ : Earth stations utilise the same frequencies and have the same capacity to damage the atmosphere.

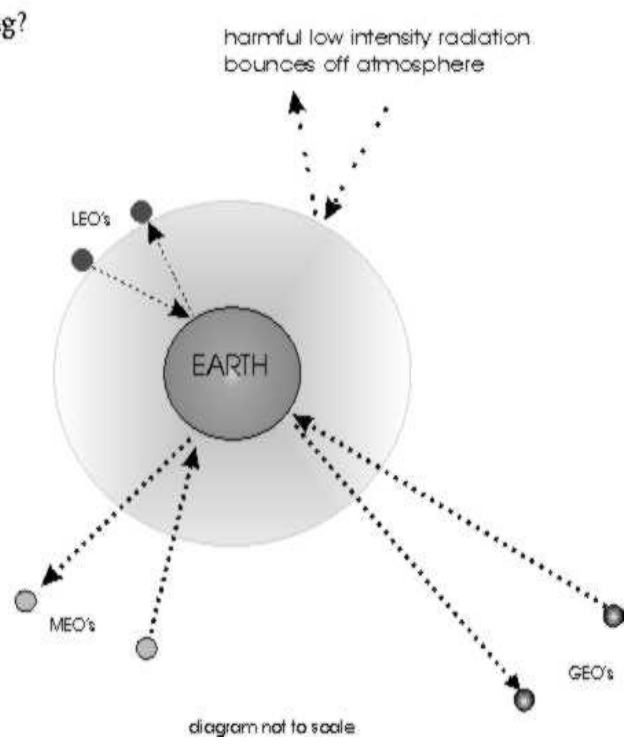

Figure 1: Microwave satellite fundamentals

The use of the two radio frequency beams to overcome atmospheric and distance issues is of concern when the radio frequency is compared to the frequency used in microwave ovens to heat and cook food. Microwave ovens work on the principle of molecular excitation that specifically targets water molecules. Bloomfield (2002) describes how the microwave energy twists and untwists the water molecule as the energy beam passes through it. As the water molecule 'dances' to the radio frequency tune, it rubs against other molecules causing friction, cooking the food. Microwave ovens use the $2450 \mathrm{MHz}(2.45$ $\mathrm{GHz}$ ) radio frequency band and the correlation between microwave oven frequency and GPS satellite transmissions may be one of the factors contributing to global warming. Our world is water-based, most organisms, including the earth, are approximately $70 \%$ water. The microwaves are heating the waterladen atmosphere, heating water on and in the earth's surface, and heating plants, animals, and humans.

Current satellite technology utilizes a higher intensity of radio frequency due to the atmospheric anomalies occurring at various levels. Leick (1995) explains that problems such as bending of the signal path caused by the Doppler effect, time delays, and advancement of carrier phases, occur due to the distance of satellites from earth, and the different characteristic of the various atmospheric layers requires more intense signals. He also indicates that ultraviolet radiation, solar flare activity and eclipses, the launch of satellites or rockets that penetrate the atmosphere, and the geomagnetic field controlling particle motion, all cause ionization that "affect the propagation of radio waves, and thus affect the signals from GPS satellites" (Leick 1995). Leick (1995) explains these specific problems occur in the ionosphere, approximately $50-1500 \mathrm{~km}$ above earth because of the presence of free - negatively charged - electrons 
which can reflect carrier signals less than $30 \mathrm{MHz}$, and only higher intensity radio frequencies can penetrate this layer. Data transmission problems occur for earth-based transmission stations when rain falls causing attenuation in frequencies above $10 \mathrm{GHz}$ (Stallings and van Slyke 1994). There could also be an environmental correlation between areas of statistically less rainfall causing drought conditions, and higher microwave radio frequency usage. Transmission interference with regards to overlapping signals has been reduced due to strict global standards (IEEE-SA Standards Board 1999, Economic Commission for Europe 2002). Satellites colliding with space junk is becoming frequent, as debris from other space voyages accumulate but meteor showers or plasma clouds created by meteors, is causing damage to telecommunication satellites (Savage and Neal 2002).

If the SMHId system fails because an isolated terminal 'crashes' or the local network goes down, and there are many reasons failure or downtime could occur, individuals become a persona non grata within the system. This issue could possibly be addressed by the sheer number of satellites surrounding, or being placed in orbit around the earth, as suggested by Considine (1995). Also, if the SMHId system misreads or is unable to read the data contained on the microchip, the SMHId recipient will not exist in the system. Having unidentifiable SMHId recipients could create a national security risk especially in light of escalating terrorist activities around the globe. Roger Clarke (2001) is one of a number of academics addressing privacy issues regarding access to personal data, which requires substantial computer and data security and the undivided attention of computer experts. This adds to the cost of transmissions, which often are passed on to the consumer, and in the light of current global economic problems, communities and nations may be unwilling to support the developing telecommunication system, making SMHId implementation, and success, doubtful.

In Australia, numerous groups and individuals have voiced their opinions regarding the benefits and consequences of microwave technology to the government, and a report was published in May 2001 (Inquiry into Electromagnetic Radiation 2001). Although many researchers contributed to the initial findings, the Senate Committee concluded that the research was not sufficiently rigorous and allocated funding for continued research. The studies were criticized because of the singular nature of each experiment but only the selected submissions were read and noted by the Committee. No previous international research was cited, therefore the Committee gave recommendations based on limited information. The level of disinformation is disturbing especially when research into microwave radio frequency extends back to the 1940's with the advent of radar technology (Considine 1995). The Committee recommended further funding to identify knowledge gaps, and replication of submitted scientific data as soon as possible due to the "economic value of the mobile phone industry, revenue earned by the government from the industry, and the large number of people exposed" (Inquiry into Electromagnetic Radiation 2001, p.xx). Overall, the report reflects other recent research findings from various countries, with most offe ring vague conclusions to current research. However, most investigative committees recommend erring on the side of caution when using microwave radio frequency (Bloomfield 2002; Center for Devices and Radiological Health 2000; Federal Communications Commission 2002; Laboratorie Central des Industries Électriques 2002; National Radiological Protection Board 2003).

\section{Impact on Health Service Industry}

Reports by the National Radiological Protection Board (2003), Lien et al (2001), and the Center for Devices and Radiological Health (2000) suggest there is weak evidence of cancer caused by radio frequency radiation, which supports the results of radio frequency exposure on research animals. However, the results obtained through animal research are based on a minimal number of scans per animal in comparison to possible utilization of a fully encompassing SMHId system for financial transactions and surveillance purposes. The possible number of scans per SMHId recipient is significantly increased due to increased satellite numbers and transmission requirements, individual movement in an area, contact with other SMHId recipients, employment or schooling security systems, access to individual or bus i- 
ness bank accounts and all other financial transactions including fuel, food, road tolls, postage, telephone calls, etc. This system description is familiar because it is a system that western society is already accustomed to with multiple card or identifier access. Also, if the system is based on microwave data transfer technology it is possible tumors, cancers, cataracts, biological effects from thermal stress, destruction of the immune system, and neurological effects from prolonged exposure may also increase (Berkow 1997, Spraycar 1995).

The Inquiry into Electromagnetic Radiation (2001) Committee found that "while adverse health effects are not agreed upon, the existence of biological effects associated with radio frequency radiation is now recognized. For these reasons the Committee Chair recommended a rigorous precautionary approach in all areas of deployment of wireless technology, and radio frequency (RF) emissions be kept As Low As Reasonably Achievable (ALARA)" (p.xv).

The report continues by discussing possible health risks based on submitted and recently published scientific data. The data presented relating to health risks included,

\begin{abstract}
"DNA damage, heat shock protein response, changes in the movement of substances across cell membranes, changes in the blood brain barrier, oncogene change, melatonin reduction and altering of calcium ion signalling. In animals studies have shown chromosome aberrations, increases in double and single strand DNA breakages, increases in the promotion of certain cancers in genetically predisposed mice, severe depression of the immunological and endocrinological responses of young chickens, changes in temperature regulation, changes to calcium ion mobility in the brains of cats and rabbits, changes to the proliferation rate of cells, alterations to enzyme and nervous system activity and behavioural change, at low level exposure to radio frequency radiation" (p.xvi).
\end{abstract}

This indicates that SMHId recipients may possibly have greater health problems. The report by the Telecommunications Technology Council (2000) of Japan, listed three types of effects caused by electromagnetic fields: thermal, stimulation, and other. Table 1 explains the relationship between electromagnetic waves and recorded physiological effects (Telecommunications Technology Council, 2000, p.33).

The physical response to the causal effects of electromagnetic radiation include;

Thermal effects - thirst, tiredness, nausea, vertigo, reduction in sweat rate, hot dry skin, muscle cramps, convulsions, coma and possible death. One in every three individuals who survive a near-fatal case of increased core temperature remains permanently disabled with multi-system organ dysfunction. The

\begin{tabular}{|c|c|c|c|c|}
\hline \multicolumn{2}{|c|}{ Effect Type } & $\begin{array}{l}\text { Factors of Physiological } \\
\text { Changes }\end{array}$ & $\begin{array}{l}\text { Quantities to be } \\
\text { Evaluated }\end{array}$ & $\begin{array}{l}\text { Relationship with Elec- } \\
\text { tromagnetic Waves }\end{array}$ \\
\hline \multirow{2}{*}{$\begin{array}{l}\text { Thermal } \\
\text { Effects }\end{array}$} & $\begin{array}{l}\text { Whole } \\
\text { body } \\
\text { heating }\end{array}$ & $\begin{array}{l}\text { Heat adjustment response } \\
\text { Rise in inner body tem- } \\
\text { perature } \\
\text { Thermal stress }\end{array}$ & $\begin{array}{l}\text { Whole body average } \\
\text { SAR * } \\
\text { Temperature increase } \\
\text { (inner body) }\end{array}$ & \multirow{3}{*}{$\begin{array}{l}\text { Macro-level interaction } \\
\text { (Dosimetric approaches } \\
\text { can be applied) }\end{array}$} \\
\hline & $\begin{array}{l}\text { Partial } \\
\text { body } \\
\text { heating }\end{array}$ & Heating of organism & $\begin{array}{l}\text { Partial body average } \\
\text { SAR } \\
\text { Temperature increase } \\
\text { (particular part of or- } \\
\text { ganism) }\end{array}$ & \\
\hline \multicolumn{2}{|c|}{ Stimulation effects } & $\begin{array}{l}\text { Excitation of nerves and } \\
\text { muscles due to electrical } \\
\text { stimulus }\end{array}$ & $\begin{array}{l}\text { Induced current (den- } \\
\text { sity) }\end{array}$ & \\
\hline \multicolumn{2}{|c|}{ Other effects } & Unknown & $\begin{array}{l}\text { Electromagnetic field } \\
\text { strength, etc (modu- } \\
\text { lated frequency) }\end{array}$ & Micro-level interaction \\
\hline
\end{tabular}

* Specific Absorption Rates

Table 1: Relationship between electromagnetic waves and physiological effects (Telecommunications Technology Council, 2000, p.33) 
symptoms are similar to heat stroke. It is important to note that humans can only tolerate a increase of core body temperature of $5^{\circ} \mathrm{C}$ and body temperatures above $43{ }^{\circ} \mathrm{C}$ can cause cellular damage resulting in permanent brain damage (Stedman's Medical Dictionary, 1995).

Stimulation effects - whether local or diffused, include muscle spasm, an internalized buzzing sensation, "seizures, possible brain hemorrhages, breathing paralysis, psychological changes, for example, shortterm memory problems, personality changes, irritability, sleep disturbances, and irregular heartbeat" (Berkow 1997). On a cellular level, or micro-level, it is possible that cell membranes could rupture damaging cells and leaking fluid, causing oedema. This can lower blood pressure, causing a loss of blood to the brain and other vital organs, with visible symptoms such as fainting, coma, and possible death.

Berkow (1997, pp. 1340-1342), and Spraycar (1995, p. 1483) both identify radiation effects as the results of shortwave radiation. They continue to explain that long term exposure to radiation or repeated exposure to low doses of radiation can have the following effects: cessation of menstruation, decreased fertility in men and women, decreased sex drive in women, cataracts, and decreased red and white blood cells, and platelets. The decline in blood cells numbers is similar to autoimmune diseases, and the human body is less able to fight infections, viruses, or bacteria when this occurs. Berkow (1997) lists the symptoms of long-term radiation exposure to include: hair loss, skin wasting, formation of open sores or ulcers, calluses, and spider veins. Decades of low doses of radiation may also cause squamous skin cancers, and bone tumors. Sun exposure and ultraviolet radiation warnings are now common events in Australia. Especially noticeable is the "Slip, Slop, Slap" summer campaign encouraging people to cover up and use sunscreen. Ultraviolet readings are also publicized during weather segments on local television and radio stations, encouraging people to lessen outdoor activity or to wear long-sleeved clothing and sunscreen minimizing sun exposure, and by coincidence, radio frequency burns.

As a comparison to the information on shortwave radiation from medical sources, Considine (1995, p.2613) explains shortwave radiation to be radio frequency in the "200 $\mathrm{MHz}$ to $35,000 \mathrm{MHz}$ range, with wave lengths of $1.5 \mathrm{~m}$ and $8.5 \mathrm{~mm}$, respectively". This corresponds to the radio frequency used in GPS satellites, therefore radio frequency used for satellite transmissions is indeed a health issue. The research on the causal effects of radio frequency for SMHId illustrates the impact this technology may have on the overall health system. There most likely will be increased pressure on the health insurance industry, public and private health systems, government health funding, and all levels of staff involved in health care. From the compiled research, the current level of technology is already producing causal effects, therefore implementation of SMHId will only serve to amplify the predicaments society is placed in.

Hindle (2000) indicates that a recent Senate inquiry found many health professionals who regard the current health system as "overburdened", as did the Australian Nursing Federation (n.d.), Ford (2002), and Friends of Medicare (2001) who indicate that "changes and reductions in government funding have played a major role" in the health care dilemma. The article concludes that minimal understanding of health system problems are due to the complexity of the system and lack of available data. SMHId implementation would seemingly add to an already overtaxed health system.

An online article by Brown (2002) who is situated at the Washington Headquarters of NASA, indicates that scientists, not wanting to sound alarmist, but concerned with the effects of global warming, explain that the increased temperature is allowing a greater number of viruses, bacteria, and disease carrying insects to develop and spread. The warning exists for all humanity. Increased microwave transmissions will increase the temperature, increase the spread of viruses and disease, lower immune response, increase health problems, and therefore place enormous pressure on health systems. 


\section{Conclusion}

The initial research by the author on this subject began in 1986, and technological advancements that were considered futuristic have come to fruition. SMHId technology has evolved from a long line of technological artefacts but the taxonomic classification of this particular technological artefact reveals weaknesses in the technological lineage (Covacio 2003). The correlations reveal weaknesses between the interaction of microwave data transfer technology, the environment, and SMHId recipients, impacting the health system. Firstly, the continual bombardment of the atmospheric layers by microwave radiation from satellite communication systems will eventually heat the atmosphere to possibly unlivable conditions. Secondly, if the SMHId system relies heavily on data transference based on GPS satellites the transmissions may eventually 'cook' SMHId recipients because of repetitive and almost constant exposure to microwave radiation. Thirdly, the actual microwaves targeting and surrounding SMHId recipients cause excitation of water molecules within the human body causing many associated health problems. Articles in medical texts, government investigative committees, and health-related web sites support this hypothesis.

The issues surrounding microwave radio frequency requires further elucidation, although research, articles and textual information from related disciplines indicate, in most instances, a high level of risk associated with this technology, therefore SMHId implementation may be hazardous to participants. If government committees, industry boards, working groups, or opinion leaders continue to support and assist the adoption of this technology, either willingly or unwittingly, widespread damage to the environment, fauna, and humans seems inevitable.

Initially it may seem this human tagging system offers many benefits, but the primary concern to SMHId investors should include the long-term social consequences. If decimation of SMHId recipients occurs, are current advocates of the technology willing to accept the long-term responsibility of the damage to the environment and people? If a SMHId system is operated in either a closed or open environment, people who choose to opt-in to the system should be clearly notified by SMHId advocates and supporting authorities of the increased health risks associated with the technology.

A proactive approach distinguishing socio-technological controversies surrounding SMHId would be expedient to government ministers, other professionals and experts, and to the people who endure the mistakes made by those who instigate social systems. The literature from numerous disciplines relating

to SMHId conception and implementation supports the concerns regarding identification technology but the high level of controversy surrounding SMHId has limited the number of academics discussing this particular technology openly. A balanced argument providing a clear understanding of the pros and cons is required, so individuals can choose whether to opt-in to the system or not. This article aims to fill a small portion of the large gap in understanding this possible emotive socio-technological situation.

\section{References}

3G-Generation. (2000). Retrieved 15 February 2003, from www.3g-generation.com

Appell, D. (2003, January). Getting under your skin: Safety questions about implantable chips persist. Scientific American, 287(1), 18-19.

Australian Communications Authority. (2000). Human exposure to radiofrequency electromagnetic energy: information for manufacturers, importers, agents, licencees or operators of radio communications transmitters. Australian Regulations. Law Courts, Melbourne.

Australian Nursing Federation: Queensland Branch. (n.d.). Healing our hospitals: Senate inquiry findings released. Media Release. Retrieved 18 February, 2003, from www.qnu.org.au/senate.htm

Berkow, R. (Ed.). (1997). The Merck manual of medical information. Merck Research Laboratories: New Jersey. 
Technological Problems with SMHId

Bloomfield, L. A. (2002). How things work: microwave ovens. University of Virginia, Physics Department. Retrieved 15 February 2003 from http://rabi.phys.virginia.edu/HTW/microwave ovens.html

Bogard, W. (1996). The simulation of surveillance: Hypercontrol in telematic societies. Cambridge University Press: Great Britain.

Brown, J. (2002). Warmer world will be a sicker world, says scientists. Earth Observatory, Media Alerts Archive, NASA, US Government. June 20. Retrieved 11 March 2003 from http://earthobservatory.nasa.government/Newsroom/MediaAlerts/2002/200206209416.html

Center for Devices and Radiological Health. (2000). Microwave oven radiation. US Food and Drug Administration. Retrieved 15 February, 2003, from www.fda.government/cdrh/consumer/microwave.html

Clarke, R. (1994). Human identification in information systems: Management challenges and public policy issues. Information Technology \&People, 7(4), 6-37.

Clarke, R. (2001). Person-location and person-tracking: Technologies, risks, and policy implications. Information Technology \& People, 14(2), 206-231.

Considine, D. M. (Ed.). (1995). Van Nostrad's Scientific Encyclopedia. $\left(8^{\text {th }}\right.$ ed.) Vol. 1 \& 2. Van Nostrand Reinhold: New York.

Covacio, Silvia. (2003). Taxonomic categorisation of technological artefacts. Working paper submitted to $16^{\text {th }}$ Bled Electronic Commerce Conference - eTransformations, Bled, Slovenia, 9 - 11, June.

Department of Health, Education and Welfare. (1973). ITG subject: diathermy. US Food and Drug Administration, May. Retrieved 15 February, 2003, from www.fda.government/ora/inspect ref/itg/itg10.html

Disability Online. (2002). Food allergy means your body reacts to certain foods. Retrieved 7 March, 2003, from www.disability.gov.au

Economic Commission for Europe (2002). An international model for technical harmonisation based on good regulatory practice for the preparation, adoption and application of technical regulations via the use of international standardstelecom industry initiative. Committee for Trade, Industry and Enterprise Development, Working Party on Technical Harmonisation and Standardization Policies, $12^{\text {th }}$ Session, United Nations. 15 October.

Economist Technology Quarterly. (2001). No hiding place for anyone. September, p. 10.

Federal Communications Commission (2002). Exposure of Salzburg. R. Coray (Coordinator). Federal Office of Communications, Austria.

Ford, Greg. (2002, October 11). Rest in peace, Medicare. The Age, Melbourne. p. 11.

Friends of Medicare. (2001). Information Kit: It works, it's fair, it's Medicare. Retrieved 18 February, 2003, from www.phaa.net.au/friends of medicare

Gerritsen, M., Kros, A., Sprakel, V., Lutterman, J.A., Nolte, R.J. \& Jansen, J.A. (2000). Biocompatibility evaluation of solgel coatings for subcutaneously implantable glucose sensors. Biomaterials, 21(1), 71-78.

Giddens, Anthony. (1993). Sociology. (2 ${ }^{\text {nd }}$ ed.). TJ Press Ltd.: Great Britain.

Gruys, E., Schakenraad, J.M., Kruit, L.K. \& Bolscher, J.M. (1993). Biocompatibility of glass-encapsulated electronic chips (transponders) used for the identification of pigs. Veterinary Record, 133(16), 385-388.

Hindle, Don. (2000). Taking health seriously: The Senate inquiry into public hospitals. Australian Health Review, 23(2), 3-9. Editorial.

IEEE-SA Standards Board. (1999). IEEE standard for safety levels with respect to human exposure to radio frequency electromagnetic fields, $3 \mathrm{kHz}$ to $300 \mathrm{GHz}$. (IEEE C95.1-1991 \& IEEE C95.1a-1998) Institute of Electrical and Electronics Engineers, Inc., New York.

Inquiry into Electromagnetic Radiation. (2001). Report of the Senate Environment, Communications, Information Technology and the Arts References Committee. (Senator Lyn Allison, Chairperson) Parliament of the Commonwealth of Australia, Canberra.

Knapp, Terry R. \& Belsey, Elisabeth Mary. (1998). Temporary implant with transponder and methods for locating and identifying. (Lipomatrix Inc.) US Patent 5725578.

Laboratoire Central des Industries Électriques. (2002). Controlling the risks of human exposure to electromagnetic fields: SAR measurement for mobile phones. France. Retrieved 15 February, 2003, from www.lcie.com 
Lambooij, E., Langeveld, N.G., Lammers, G.H. \& Huiskes, J.H. (1995). Electronic identification with injectable transponders in pig production: results of a field trial on commercial farms and slaughterhouses concerning injectability and retrievability. Veterinary Quarterly, 17(4), 118-123.

Leick, Alfred. (1995). GPS satellite surveying. ( $2^{\text {nd }}$ ed.). John Wiley and Sons, Inc.: New York.

Lein, Ryan, Quinlan, Mike, Brewer, Jeff, Florez, Eric, \& Rhoden, Sam. (2001). Electromagnetic fields and applications: Cellular phone antenna design. Department of Electrical Engineering, College of Computing Sciences \& Engineering, University of North Florida. Retrieved 24 February, 2003, from http://antares.cocse.unf.edu/projects/class/spring2001

Quan, Margaret. (1999). Biochips: From sci-fi to critical tech. Electronic Engineering, 1093, 8.

Marsh, Mike. (Ed.). (2003). Transponder News @ transpondernews.com. Retrieved 15 February, 2003, from http://rapidttp.com/transponder/index.html

National Institute of Allergy and Infectious Diseases (2002). Allergy statistics: Fact sheet. Office of Communications and Public Liaison, National Institutes of Health, US Government. Retrieved 7 March, 2003, from www.niaid.government/factsheets/allergystat.htm

National Radiological Protection Board. (2003). Understanding radiation: Ionising radiation and how we are exposed to it. European Union. Retrieved 17 February, 2003, from www.nrpb.org

Park, Dean \& Wieser, John. (1998). Summary of field studies evaluating the efficacy of Bio-Bond, a porous polymer sheath, on radio frequency identification (RFID) transponders to prevent migration from a known implant site. Destron Fearing Corporation, US. Retrieved 13 February, 2003, from http://destronfearing.com/elect/report.html

Pugh, Angela M. (1998). Silicon implants. Data Communications, 27(14), 11.

Rao, G.N. \& Edmondson, J. (1990). Tissue reaction to an implantable identification device in mice. Toxicologic Pathology, $18(3), 412-416$.

Rossing, W. (1999). Animal identification: introduction and history. Computers and Electronics in Agriculture, 24(1-2), 1-4.

Savage, Donald \& Neal, Nancy. (2002). NASA prepares for meteor shower. SpaceLink, NASA Washington Headquarters, US Government. Release: 02-221. Retrieved 11 March, 2003, from http://spacelink.nasa.gov

Sorensen, M.A., Buss, M.S. \& Tyler, J.W. (1995). Accuracy of microchip identification in dogs and cats. Journal of the American Veterinary Medical Association, 207(6), 766-767.

Smith, C.D., Kildishev, A.V., Nyenhuis, J.A., Foster, K.S. \& Bourland, J.D. (1999). Interactions of magnetic resonance imaging radio frequency magnetic fields with elongated medical implants. $44^{\text {th }}$ Annual Conference on Magnetism and Magnetic Materials, San Jose, California, 6188-6190.

Spraycar, Marjory. (Ed.). (1995). Stedman's medical dictionary. $\left(26^{\text {th }}\right.$ ed.). Williams and Wilkins: Baltimore, MD.

Stallings, William and van Slyke, Richard. (1994). Business data communications. (2 ${ }^{\text {nd }}$ ed.). Macmillan Publishing Company: New Jersey.

Telecommunications Technology Council. (2000). Measurement of SAR from mobile phone terminals and other terminals that are intended for use in close proximity to the side of the head. Deliberation No. 118. Japan Government. Retrieved 4 March, 2003, from www.tele.soumu.go.jp

Teledesic (2002). Retrieved 15 February, 2003, from www.teledesic.com

Tomljanovic, Charles, Wright-Walters, Maxine, \& Stephensky, Jules. (1997). Anthropogenic electromagnetic fields and cancer: a perspective. Working paper. Retrieved 24 February, 2003, from www.piercelaw.edu/risk/vol8/summer/Tomljan+.htm

Weber, David O. (2000). The bionic century. Health Forum Journal, 43(3), 14-19.

Woolcock Institute of Medical Research. (2002). Facts. Retrieved 7 March, 2003, from www.irm.usyd.edu.au/facts.htm

\section{Biography}

Silvia Covacio (covacio@iprimus.com.au) is a researcher and casual lecturer at University of South Australia in the School of Accounting and Information Systems, Adelaide, South Australia. 